[Frontiers in Bioscience 13, 1646-1653, January 1, 2008]

\title{
Genetics and development of the optic chiasm
}

Eloisa Herrera, Cristina Garcia-Frigola

Instituto de Neurociencias de Alicante, Universidad Miguel Hernandez-CSIC, Campus de San Juan, San Juan de Alicante, Alicante 03550, Spain

\section{TABLE OF CONTENTS}

1. Abstract

2. Introduction: Structure, function and evolution of the optic chiasm

3. Anatomical alterations of the optic chiasm

4. Development of the optic chiasm

5. Molecular Mechanisms underlying the development of the optic chiasm

5.1. Patterning of the ventral diencephalon and the retina during early development

5.1.1. The Sonic Hedgehog (Shh) signaling cascade

5.1.2. Homeodomain proteins

5.1.3. Winged Helix transcription factors

5.2. Axon guidance at the chiasmatic midline

6. Prospective

7. Acknowlegments

8. References

\section{ABSTRACT}

In animals with binocular vision, retinal fibers either project across the midline or they remain on the same side of the ventral diencephalon, forming an X-shaped commissure known as the optic chiasm. The correct formation of the optic chiasm during development is essential to establish a fully functional visual system. Visual dysfunction associated with axonal misrouting at the optic chiasm has been described in albino individuals and in patients with non-decussating retinal-fugal fiber syndrome. Although little is known about the causes of retinal misrouting in these conditions, the molecular mechanisms responsible for the formation of the optic chiasm are beginning to be elucidated in vertebrates. This review focuses on our current knowledge of how the optic chiasm forms, which will hopefully help us to better understand these congenital anomalies.

\section{INTRODUCTION: STRUCTURE, FUNCTION AND EVOLUTION OF THE OPTIC CHIASM}

In binocular organisms, retinal ganglion cell (RGC) axons from the nasal hemiretina generally cross the brain midline and project to their visual targets in the contralateral brain hemisphere, the lateral geniculate nucleus (LGN) and the superior colliculus. However, some fibers from the temporal retina do not cross the midline but rather they project to targets on the same side. This axonal hemidecussation takes place at the ventral diencephalon midline, in a structure known as the optic chiasm. The main function of the optic chiasm is to organize the inputs from the same point in the visual field perceived by each retina, directing these to the same location in the visual cortex. It is there, in the visual cortex, that the two subtly different images are integrated into a cohesive three-dimensional representation (1): Figure 1). 


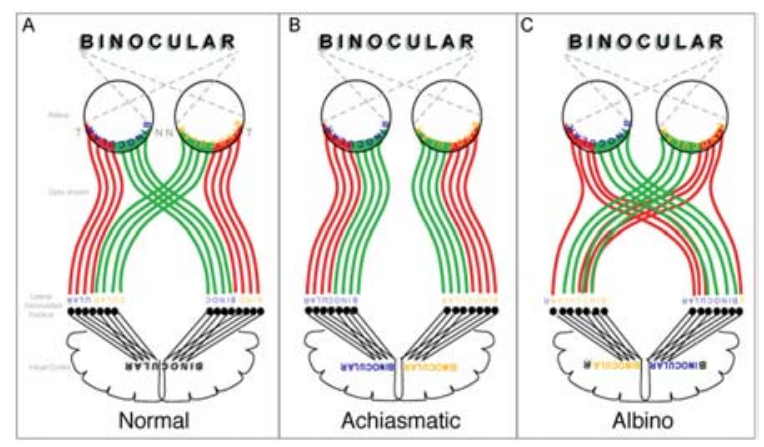

Figure 1. Extreme phenotypes caused by the misrouting of retinal fibers at the optic chiasm. A. In primates, the nasal retinal axons (green) cross the midline at the optic chiasm while temporal retinal axons (red) extend ipsilaterally, projecting toward the lateral geniculate nucleus and then the visual cortex, maintaining the strict retinotopic segregation of the visual field. B. In achiasmatic individuals (non-decussating retinal-fugal fiber syndrome) nasal retinal fibers fail to decussate adequately at the chiasm, instead projecting ipsilaterally towards the lateral geniculate nuclei together with temporal retinal fibers. C. In albinism, ipsilaterally destined temporal retinal fibers erroneously decussate and project contralaterally at the optic chiasm. In both of these conditions, the corresponding visual field is a complete (achiasmatic) or partial (albinism) mirror inversion of the representation in each hemisphere. In most cases, these individuals with an aberrant ipsi/contralateral ratio develop congenital nystagmus. $\mathrm{N}$, nasal retina, $\mathrm{T}$, temporal retina.

In different species, the number of fibers that do not cross the midline at the optic chiasm is proportional to the size of the binocular visual field, which in turn depends on the laterality of the position of the eyes in the head. Partial decussation of the fibers from each eye occurs in most mammals with frontally placed eyes, such as humans or non-human primates, in which the uncrossed component reaches nearly $40 \%$ of all RGCs (2), (3), (4). The heads of cats, dogs, ferrets, rabbits or mice have differing degrees of eye separation and they show a variation in the uncrossed component from $30 \%$ in cats to about $5 \%$ in mice, the eyes of which are situated laterally and that have poor binocular vision (5). Amphibians such as Xenopus are interesting since a subpopulation of ganglion cells in the ventrotemporal (VT) retina begin to project ipsilaterally at metamorphosis, when the laterally located eyes become positioned frontally (6), (7). Birds and fish have lateral eyes and panoramic vision, and except for some ipsilateral axons that disappear at late stages of development, they do not develop an uncrossed projection (8), (9), (10), (11).

\section{ANATOMICAL ALTERATIONS OF THE OPTIC CHIASM}

In humans, two conditions involve extreme abnormalities of the optic chiasm: albinism and achiasmia (or non-decussating retinal-fugal fibre syndrome, NDRFS). In albinism, a significant number of temporal retinal fibers erroneously decussate and project contralaterally. However, in NDRFS, nasal retinal fibers fail to decussate adequately at the chiasm, projecting instead ipsilaterally towards the lateral geniculate nuclei along with temporal retinal fibers. In both cases, the corresponding visual field is a completely (achiasmatic) or partially (albinism) reversed in the laminae of the lateral geniculate nuclei (Figure 1). In general, individuals with an aberrant ratio of ipsilateral/contralateral projections (both albino as well as NDRFS patients) show classic congenital nystagmus in the horizontal plane, and/or see-saw nystagmus in the vertical plane (12). Thus, there appears to be a causal association between misrouting at the optic chiasm and nystagmus. In fact, given that see-saw nystagmus is also displayed by a well-documented family of achiasmatic mutant Belgian sheep dogs (13), achiasmia was proposed to be sufficient for the development of congenital see-saw nystagmus (14). More recently, in the achiasmatic belladona zebrafish mutants identified in a behavioral screen, congenital nystagmus has been described as a consequence of the misrouting of retinal axons to the ipsilateral side of the brain (15).

Several attempts to rescue the albino phenotype have been made. The Tyrosinase gene, is known to be altered in a particular type of inheritable albinism in humans, oculocutaneous albinism type I. When this gene is expressed in the retinas of albino mice at early developmental stages it rescues the retinal axon projection phenotype at the optic chiasm (16) suggesting that tyrosinase plays an important role in optic chiasm development. However, spatiotemporal aspects of neurogenesis are also altered in albino mice (17) and it has been proposed that such alterations could influence the specification of RGCs. Some studies have associated the lack of DOPA, a melanin precursor, with alterations in the cell cycle of retinal cells (18), (19). In turn, this could affect the differentiation and specification of different RGC populations, although further experiments will be needed to fully understand the mechanisms relating the melanin pathway with retinal axon misrouting at the midline.

\section{DEVELOPMENT OF THE OPTIC CHIASM}

Because of its many advantages as an animal model, the mouse is the most commonly used mammal to study both the decussation of retinal axons at the midline, as well as other aspects of optic chiasm development. The formation of the optic chiasm in the mouse has been seen to occur in three sequential steps. In a first phase, pioneer axons from the dorso-central retina navigate into the optic stalk to enter the hypothalamic area. The cellular environment of the developing diencephalon is comprised of radial-glial cells that have been shown to contact both ipsilateral and contralateral RGC axons. On the other hand, there is a neuronal population that appears in the ventral diencephalon prior to the arrival of any retinal axons. This early population of neurons express the carbohydrate antigen SSEA-1, and they are distributed in a characteristic $\mathrm{V}$-shape that defines the midline and posterior border of the future chiasm (20). The majority of the pioneer axons cross the midline and grow in close association with the array of early SSEA-1 expressing neurons, establishing the position of the optic chiasm along the anterior-posterior axis of the brain. A small population of these axons extends 


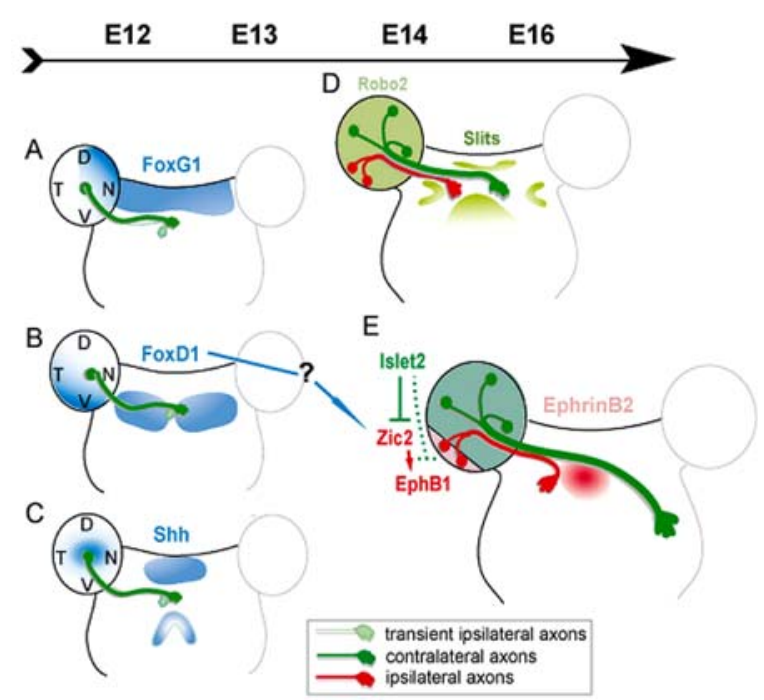

Figure 2. Schematic diagrams depicting the retinal axon pathways in relation to the patterns of regulatory genes and axon guidance molecules expressed during the development of the optic chiasm. Left panels show the early stages of optic chiasm formation in relation to the patterning of the retina and ventral diencephalon. Right panels show the later stages of optic chiasm formation in relation to retinal axon guidance. A. FoxG1 is expressed in progenitor cells in the nasal retina and in the dorsal diencephalon. B. FoxD1 is expressed in the VT retina, in a pattern complementary to FoxG1 and in the future chiasmatic region when it forms two bilateral patches at both sides of the midline where the optic chiasm forms. C. Sonic Hedgehog (Shh) is expressed early in the retina in a central-to-peripheral gradient. This expression is important for RGC differentiation. Later, Shh is also expressed in the diencephalon helping to guide axons through repellent signals. D. Around E14, Slit proteins are expressed in the chiasm area forming a X-shaped corridor that is repulsive for all RGCs that express the Robo2 receptor. E. Axonal divergence takes place when RGCs fibers from the VT retina, expressing the transcription factor Zic2 (in the same cascade as FoxD1 and apparently repressed by Islet2) and the receptor EphB1, reach the midline. These axons are inhibited by ephrinB2 that is expressed by a small population of glial cells at the midline. D, dorsal, N, nasal, $\mathrm{V}$, ventral, T, temporal. E12-16, embryonic day 12 to 16 . ipsilaterally and forms an early-uncrossed projection that is thought to be transient since it is not detected at later stages.

A second phase commences around E14, and involves the arrival at the midline of retinal axons from the VT crescent in the retina and while these turn back to the same side of the brain from which they originate, the axons from the rest of the retina cross the midline (21), (22). The arrival of the VT axons at the midline is accompanied by a short pause and dynamic changes in the growth cone that enable it to recognize inhibitory cues at the optic chiasm midline (23). The ipsilateral axons from the peripheral VT retina generated in this second phase constitute the permanently uncrossed component (Figure 2). In the third and final phase, no RGCs establish uncrossed projections and thereafter, all ganglion cells extend a crossed projection whether they are born in the VT segment or not (24).

Thus, it is interesting to speculate that the time of ganglion cells generation, and hence, the arrival of their axons to the chiasm is important in the selection of the pathway. Indeed, an interesting phenomenon that influences retinal axon decussation at the optic chiasm is the relationship between the fibers from the two eyes. It is well known that early monocular enucleation in rodents reduces the ipsilateral projection of the contralateral eye, mimicking the albino phenotype. Axons from the VT retina are lost when they reach the midline and thus, the interaction between axons from both eyes is essential for axon routing at the optic chiasm. However, this effect does not seem to be valid for other mammals such as marsupials. Such differences can be explained by the relative location of the uncrossed component in the optic chiasm. While in rodents ipsilateral axons approach the midline, in marsupials they remain laterally segregated and they do not interact with the midline. Interestingly, recent findings suggest that humans would share the organization of marsupials rather than that of rodents (25).

All these sequential processes are the result of a combination of tissue patterning events and axon guidance decisions taken by RGC axons at the ventral diencephalic midline. Although patterning and axon guidance take place in an inseparable manner, in the following sections we shall first analyze the mechanisms behind the patterning of the retina and the ventral diencephalon, before going on to discuss the molecular basis underlying specific axon guidance responses.

\section{MOLECULAR MECHANISMS UNDERLYING THE DEVELOPMENT OF THE OPTIC CHIASM}

\subsection{Patterning of the ventral diencephalon and the retina during early development}

A number of proteins involved in the morphogenesis of the midline are expressed in the area of the future optic chiasm early in development, and play a critical role in the patterning of the ventral diencephalon:

\subsubsection{The Sonic Hedgehog (Shh) signaling cascade}

In the chick, Shh is present along the entire axial midline of the embryo prior to the arrival of the retinal axons. As the first retinal axons approach this area, Shh is specifically downregulated at the level of the optic recess (Figure 2), a spatiotemporal change that unblocks the extension of retinal axons (26). In Pax2 null mice and in the equivalent noi zebrafish mutant, the persistent expression of Shh in the optic recess prevents the chiasm from developing (27), (28). Thus, the dowregulation of Shh expression in the region of the optic recess area is highly dependent on patterning events directed by Pax 2 . Both Pax2 and Shh are expressed at early stages of retinal development but in this context, it seems that the Shh pathway controls the expression of Pax2, contributing to convert the optic vesicle into the optic cup (29), (30), (31). Mutations in $\operatorname{Pax} 2$ can cause nerve coloboma, whereas 
mutations in Shh are associated with retinal coloboma and holoprosencephaly (32), as a consequence of its role in midline development and neural tube differentiation.

Moreover, mutations in the zebrafish Gli2 gene (the you-too or yot mutants), a Hedgehog target, produce alterations in the proper patterning of the midline glia. Such mutations also impair the expression of Slits and Sema3D, two axon guidance molecules that are important for axon navigation in the chiasmatic region (see below). As a consequence, the yot mutants develop an achiasmatic phenotype (33).

\subsubsection{Homeodomain proteins}

The transcription factor $\operatorname{Vax} 2$ is a homeobox protein whose expression is confined to the ventral region of the prospective neural retina (34). Over-expression of this gene at early stages of Xenopus and chicken development, together with loss-of function studies in the mouse, have identified a role for this protein in the specification of the ventral retina. Since ipsilateral RGCs are located in the ventral retina, it is reasonable to think that alterations in the expression of Vax 2 might affect the formation of the ipsilateral projection. Although two different groups have explored this issue, the identification of an ipsilateral component in a Vax2 mutant comparable to that of wild-type littermates (35) failed to confirm the early report of the complete loss of ipsilateral axons in Vax2 knockout mutants (36). Thus, the effect of the ablating Vax 2 on the formation of the optic chiasm must be further studied.

The LIM homeodomain transcription factor Lhx2 is expressed during the early morphogenesis of the ventral diencephalon in zebrafish. Mutations in this protein (the belladona mutants) cause an achiasmatic phenotype, although the origin of this axonal misrouting is not clear. Nevertheless, this phenotype could once again be at least partially explained by alterations in the expression of the axon guidance molecules Sema3D and Slit2 in the diencephalon of this mutant (37).

\subsubsection{Winged Helix transcription factors}

FoxD1 and FoxG1 are other proteins that affect early patterning of the retina and the chiasm (Figure 2). These two proteins from the forkhead family of transcription factors are expressed in adjacent domains of the early optic cup and they are important for establishing polarity along the naso-temporal axes of the mature retina (Yuasa et al., 1996). Altering the expression of these two genes in the retina affects the expression of downstream molecules known to influence axonal navigation along the retinofugal pathway (see below, (38); (39); (40). In addition, this pair of transcription factors are expressed in opposing rostro-caudal domains in the region of the ventral diencephalon, where the optic chiasm forms (41). While the FoxD1 domain encompasses the region that early retinal axons traverse to establish the optic chiasm, FoxG1 is located more rostrally. Interestingly, genetic ablation of FoxD1 in the mouse causes misexpression of FoxG1, accompanied by aberrant expression of Slit2 and misrouting of the retinal fibers at the chiasm (38). Thus, the ipsilateral projection augments in FoxG1 null mice (42). Because both of these genes are expressed in the retina and the diencephalon during optic chiasm development, further studies will be needed to dissect out their specific contributions at each stage of visual system development.

The correct patterning of the ventral midline also appears to be influenced by the formation of the dorsal midline. The combined influence of dorsal and ventral inductive signals has been demonstrated in the correct formation of the spinal cord (43), (44). In this structure, failure to close the neural tube has been associated with perturbed patterning at the optic chiasm in several mutant mouse lines, including Lp (loop-tail), Pax3 and Crc (circle tail). This is associated with an increase in the ipsilateral projection originated through the expansion of RGCs in the VT retina (45). Eye development begins with the evagination of the optic primordia from the diencephalic area of the forebrain. Concomitantly, the prospective optic chiasm region forms at the ventral diencephalon. Thus, it is quite possible that genes that are important for early diencephalic patterning may affect later morphogenesis of the retina and the optic chiasm. In turn, these alterations may influence the proper expression of axon guidance molecules that are essential for the navigation of retinal axons at the optic chiasm midline.

\subsection{Axon guidance at the chiasmatic midline}

As well as its critical role in visual function, the partial decussation of retinal axons at the optic chiasm has traditionally represented a good model to investigate the molecular basis of axon guidance at the mammalian midline. The molecular elements underlying the phenomenon of midline crossing were first dissected out in the Drosophila ventral nerve cord and in the vertebrate spinal cord (reviewed by (46). Both systems use the same general mechanism to control axon crossing at the ventral midline, which essentially involves the secretion of attractants such as Netrins, and that of repellents such as Slits that are expressed in a stripe along the midline. Axons modulate their responsiveness to these two signals as they grow and crossing axons that express the Netrin-receptor DCC (deleted in colorectal carcinoma) are initially attracted to Netrin and insensitive to Slits. The net effect of this phenotype drives these axons to the midline and after reaching the midline, they become insensitive to Netrin and they upregulate Robo2, a receptor for Slit, which propels them out of the midline. This acquired sensitivity to Slit also prevents later re-crossing. In contrast, the axons destined to project ipsilaterally express Robo 2 from the outset, thereby avoiding crossing the midline (47), (48), (49), (50), (51).

Given the similarities between the fly and vertebrate ventral midlines, one might think that the optic chiasm would use the same mechanism. However, the optic chiasm is not a real ventral midline structure, as it arises from the rostral end of the embryonic neural tube. Thus, it is not surprising that it differs from the ventral nerve cord and spinal cord, both in terms of the expression of guidance signals and in how axon crossing is controlled. In the first place, Netrin is not expressed in a midline stripe at the 
chiasm and therefore, it cannot function as a midline attractant. Instead, Netrin is strongly expressed at the optic nerve head and it acts as an attractant for retinal axons that exit the retina ((52)). Secondly, Slits are not expressed in midline stripes but rather in bands that lie perpendicular to the midline. Moreover, Robo 2 is expressed by all the RGC rather than by a restricted ipsilateral or contralateral subpopulation. Functional experiments in mouse and zebrafish have demonstrated that Slit/Robo signaling does not act as the midline gatekeeper in the chiasm but rather, it defines the precise position along the midline neuroaxis at which the optic chiasm develops, as well as delineating a repulsion-free corridor that keeps the axons running inside (53), (54), (55), (56): Figure 2). Heterozygous loss of Robo in humans has been recently described and such individuals suffer dyslexia, probably due to defects of the midline commissure. Thus, it would be interesting to characterize the morphology of the optic chiasm in these individuals (57).

After realizing that Robo/Slit signaling was not the key element in the divergence of retinal axons at the midline, the search for other molecules began. The members of the family of tyrosine-kinases ephrins and their Eph receptors were good candidates, as this superfamily of immunoglobulins is made up of membrane proteins that frequently mediate repulsion. Pioneering work demonstrated that ephrinB's are directly implicated in laterality at the Xenopus chiasmatic midline (58). EphrinB is present at the chiasm during the time window in which the uncrossed component forms at metamorphosis, and premature miss-expression of ephrinB2 in the ventral diencephalon induces an ectopic ipsilateral projection (58).

Later studies demonstrated that ephrinB2 is also prominent in directing divergence at the mouse optic chiasm. The uncrossed RGC axons from the VT retina are especially sensitive to ephrinB2, inhibiting their extension through the midline (59). The discovery of ephrinB2 as a mediator of inhibition of uncrossed axons at the chiasm midline encouraged the search for the specific receptor involved in this process. Of all the possible Eph receptors that could interact with ephrinB2, only EphB1 is highly expressed in RGCs from the VT retina at the time when ipsilateral axons are turning at the midline (Figure 2). Furthermore, in mice lacking EphB1, the ipsilateral retinal projection is severely impaired, indicating that this receptor is required for the formation of the uncrossed projection (59). Thus, ephrinB2 in the chiasm and EphB1 in the retina appear to be the principle axon guidance molecules that direct retinal axon divergence at the chiasm midline (60). Interestingly, EphB1 is expressed throughout the temporal retina in humans, consistent with the fact that the projections from the entire temporal retina remain ipsilateral. Additionally, ephrinB2 is expressed at the midline during optic chiasm formation (61). These findings strongly suggest a conserved role for ephrin-B/EphB signaling in controlling ipsilateral routing of axons at the optic chiasm in humans.

But what controls the spatiotemporal specificity of expression of this axon guidance receptor in the retina?
The key component seems to be Zic2, a zinc finger transcription factor that was isolated from the mouse cerebellum and that is involved in early neural patterning. Zic2 has been described as the first regulatory protein directly involved in axon divergence at the optic chiasm (62). This transcription factor is expressed post-mitotically in RGCs that project ipsilaterally during axonal extension through the optic chiasm but not in RGCs that project contralaterally (Figure 2). Zic2 is crucial for directing the ipsilateral retinal projection, since genetically modified mice expressing low levels of this protein (Zic2 knockdown mice) show a severe reduction in the number of uncrossed axons. Zic2 expression in the VT retina is conserved in a manner that precisely mirrors the extent of binocularity through evolution. Indeed, the number of cells expressing Zic2 in the VT retina is greater in the ferret than in the mouse, consistent with the higher proportion of uncrossed axons. In Xenopus, Zic2 expression is upregulated at metamorphosis, coinciding with the late development of the uncrossed component. While in the chick and zebrafish that lack an ipsilateral projection, Zic2 is not expressed in the retina (62), (37). Moreover, in albino mice where the size of the ipsilateral component is reduced when compared to pigmented mice, as in humans, there are also fewer Zic2expressing RGCs, arguing that specification of the ipsilateral RGCs in the albino is altered.

Despite all these findings, the role of Zic2 in mediating divergence at the midline is still not completely clear. The alteration of Zic2 expression in retinal explants in vitro is sufficient to change the response of RGC neurites to cues provided by chiasm cells, indicating that Zic2 acts primarily in the retina. However, Zic2 is expressed in the presumptive chiasm and eyecup early in development before the uncrossed pathway forms. Mutations in Zic2 produce holoprosencephaly, a condition in which both halves of the forebrain are fused and that is occasionally associated with cyclopia in humans (63). Zic2 mutants also show defects in neural tube closure and it is possible that the axonal phenotype observed in the Zic2 hypomorphic mice is a consequence of its expression during the early morphogenesis of the visual system rather than a more specific role in retinal axon guidance.

Zic2 expression in the VT retina matches the spatiotemporal expression of EphB1 (64). However, despite the close relationship between $\mathrm{Zic} 2$ and EphB1, it remains to be tested whether Zic2 regulates EphB1 expression in VT retina or if it is simply expressed in a parallel program. Nevertheless, it is worth noting, that the absence of FoxD1 in the retina leads to the disappearance of Zic2 and EphB1 expression, suggesting that these three proteins are part of the same cascade (38). Moreover, there are more ipsilaterally projecting axons in mutant mice for the LIM homeodomain transcription factor Islet2, which is only expressed in contralateral but not ipsilateral RGCs (Figure $2)$. This defect is associated with an increase in the expression of both Zic2 and EphB1 (64), again suggesting a link between these two proteins. Still, several issues related to the molecular program of the ipsilateral projection remain to be resolved. For instance, it is the axon repulsive response mediated by EphB/ephrinB signaling sufficient 
for the correct formation of the ipsilateral projection? Or alternatively, are other guidance proteins also needed, such as attractive signals coming from the optic tract? If other guidance signals are required, will they also be regulated by Zic2? Finally, what are the transcriptional mechanisms by which Zic2 regulates the EphB1 receptor in such as highly regulated tissue specific pattern?

Recent reports have also identified molecules whose function is to promote midline crossing in an independent manner, not related to the ephrinB2/EphB1 signaling described for the uncrossed pathway. The cell adhesion molecule PSA-NCAM is expressed by RGC axons as well as by neuronal populations at the optic chiasm, and it seems to be crucial for the re-organization or sorting of axons at the midline. The downregulation of PSA-NCAM expression in RGC axons when they reach the chiasm is thought to reduce the degree of fasciculation and facilitate the turning of uncrossed axons at the midline (65). Similarly, the cell adhesion molecule NrCAM is expressed by RGCs that project contralaterally as well as by chiasm cells, and its disruption induces an increase in the ipsilateral projection caused by the misrouting of late born RGCs from the VT segment that would normally project contralaterally (66). All these findings support a role for this family of cell adhesion molecules in axonal crossing, probably related to the mechanisms involved in the fasciculation of crossed axons.

\section{PROSPECTIVE}

In conclusion, much progress has been made in identifying the mechanisms underlying the formation of the optic chiasm during vertebrate development. Some of the transcription factors important for the regionalization of the territory where the optic chiasm forms have been identified. Indeed, the Shh, Gli, Vax2, Lhx2 or Fox proteins all play critical roles in both the morphogenesis of the retina and the regionalization of the optic chiasm region, generating the correct expression of axon guidance molecules required for retinal axons to navigate at the midline. Alterations in any of these proteins frequently cause aberrant optic chiasm phenotypes. In addition, our knowledge of the molecular profiles that encode the uncrossed retinal pathway has improved substantially. Other molecular mechanisms involved in the postranslational regulation of axon guidance molecules not described in this review, such as the function of proteoglycans or metalloproteases may also affect retinal fiber navigation at the midline, although it seems clear that the transcription factor Zic2 and EphB1/ephrinB2 signaling are the major determinants of the uncrossed pathway. Furthermore, it appears that Islet2 plays a role in repressing this ipsilateral program. Many of these molecules and mechanisms are conserved across evolution and thus, it will be interesting to investigate whether or not mutations in the human homologues of these proteins are related to the formation of the optic chiasm and in turn to the extent of binocularity.

\section{ACKNOWLEDGMENTS}

We thank Angel Barco for critically reading of this manuscript. The work in the laboratory of E.H. is supported by the Human Frontiers Science Program (CDA2004-023), the Spanish Ministery of Science and Education, and the regional government, "Generalitat Valenciana".

\section{REFERENCES}

1. P. O. Bishop and J. D. Pettigrew: Neural mechanisms of binocular vision. Vision Res, 26(9), 1587-600 (1986)

2. L. M. Chalupa and B. Lia: The nasotemporal division of retinal ganglion cells with crossed and uncrossed projections in the fetal rhesus monkey. J Neurosci, 11, 191202 (1991)

3. Y. Fukuda, H. Sawai, M. Watanabe, K. Wakakuwa and K. Morigiwa: Nasotemporal overlap of crossed and uncrossed retinal ganglion cell projections in the Japanese monkey (Macaca fuscata). J Neurosci, 9(7), 2353-73 (1989)

4. J. Stone, J. Leicester and M. S. Sherman: The nasotemporal division of the monkey's retina. J. Comp. Neurol., 150, 333-348 (1973)

5. G. Jeffrey: Architecture of the optic chiasm and the mechanisms that sculpt its development. Physiol Rev, 81(4), 1393-414 (2001)

6. S. G. Hoskins and P. Grobstein: Development of the ipsilateral retinothalamic projection in the frog Xenopus laevis. II. Ingrowth of optic nerve fibers and production of ipsilaterally projecting retinal ganglion cells. J Neurosci, 5(4), 920-9 (1985)

7. F. Mann and C. E. Holt: Control of retinal growth and axon divergence at the chiasm: lessons from Xenopus. Bioessays, 23(4), 319-326 (2001)

8. U. Drenhaus and G. Rager: Organization of the optic chiasm in the hatched chick. Anat Rec, 234, 605-617 (1992) 9. D. D. O'Leary, C. R. Gerfen and W. M. Cowan: The development and restriction of the ipsilateral retinofugal projection in the chick. Brain Res, 312, 93-109 (1983)

10. S. L. Polyak: The Vertebrate Visual System. U. Chicago Press, Chicago (1957)

11. S. Thanos, F. Bonhoeffer and U. Rutishauser: Fiberfiber interaction and tectal cues influence the development of the chicken retinotectal projection. Proc Natl Acad Sci U $S$ A, 81, 1906-1910 (1984)

12. P. Apkarian and L. J. Bour: See-saw nystagmus and congenital nystagmus identified in the non-decussating retinal-fugal fiber syndrome. Strabismus, 9(3), 143-63 (2001)

13. R. W. Williams, D. Hogan and P. E. Garraghty: Target recognition and visual maps in the thalamus of achiasmatic dogs. Nature, 367, 637-639 (1994)

14. L. F. Dell'Osso, R. W. Williams, J. B. Jacobs and D. M. Erchul: The congenital and see-saw nystagmus in the prototypical achiasma of canines: comparison to the human achiasmatic prototype. Vision Res, 38(11), 1629-41 (1998) 15. Y. Y. Huang, O. Rinner, P. Hedinger, S. C. Liu and S. C. Neuhauss: Oculomotor instabilities in zebrafish mutant belladonna: a behavioral model for congenital nystagmus 
caused by axonal misrouting. $J$ Neurosci, 26(39), 9873-80 (2006)

16. A. Lavado, G. Jeffery, V. Tovar, P. de la Villa and L. Montoliu: Ectopic expression of tyrosine hydroxylase in the pigmented epithelium rescues the retinal abnormalities and visual function common in albinos in the absence of melanin. J Neurochem, 96(4), 1201-11 (2006)

17. R. A. Rachel, G. Dolen, N. L. Hayes, A. Lu, L. Erskine, R. S. Nowakowski and C. A. Mason: Spatiotemporal features of early neuronogenesis differ in wild-type and albino mouse retina. J Neurosci, 22(11), 4249-63. (2002)

18. M. Ilia and G. Jeffery: Retinal mitosis is regulated by dopa, a melanin precursor that may influence the time at which cells exit the cell cycle: analysis of patterns of cell production in pigmented and albino retinae. J Comp Neurol, 405(3), 394-405 (1999)

19. I. Kralj-Hans, M. Tibber, G. Jeffery and P. Mobbs: Differential effect of dopamine on mitosis in early postnatal albino and pigmented rat retinae. $J$ Neurobiol, 66(1), 47-55 (2006)

20. C. A. Mason, D. Baird, M. Morrison, S. Ward and Q. Zhang: Axon-target interactions in the developing cerebellum. Persp. Devel. Neurobiol. The Cerebellum: A model for construction of a cortex, C. Mason and C. Sotelo, eds., 5, 69-82 (1997)

21. R. W. Guillery: A comparison of eutherian and marsupial optic chiasms: A brief review. Rev Brasil Biol, 55 (Supl. 1), 1-10 (1995)

22. C. A. Mason and D. W. Sretavan: Glia, neurons, and axon pathfinding during optic chiasm development. Curr Opin Neurobiol, 7(5), 647-653 (1997)

23. P. Godement and C. A. Mason: Guidance of retinal fibers in the optic chiasm. Perspectives on Developmental Neurobiology, 1(4), 217-25 (1993)

24. U. C. Drager: Birth dates of retinal ganglion cells giving rise to the crossed and uncrossed optic projections in the mouse. Proc R Soc Lond B Biol Sci, 224(1234), 57-77. (1985)

25. M. M. Neveu, G. E. Holder, N. K. Ragge, J. J. Sloper, J. R. Collin and G. Jeffery: Early midline interactions are important in mouse optic chiasm formation but are not critical in man: a significant distinction between man and mouse. Eur J Neurosci, 23(11), 3034-42 (2006)

26. F. Trousse, E. Marti, P. Gruss, M. Torres and P. Bovolenta: Control of retinal ganglion cell axon growth: a new role for Sonic hedgehog. Development, 128, 39273936 (2001)

27. R. Macdonald, J. Scholes, U. Strahle, C. Brennan, N. Holder, M. Brand and S. W. Wilson: The Pax protein Noi is required for commissural axon pathway formation in the rostral forebrain. Development, 124(12), 2397-408 (1997)

28. M. Torres, E. Gomez-Pardo and P. Gruss: Pax2 contributes to inner ear patterning and optic nerve trajectory. Development, 122(11), 3381-3391 (1996)

29. C. Chiang, Y. Litingtung, E. Lee, K. E. Young, J. L. Corden, H. Westphal and P. A. Beachy: Cyclopia and defective axial patterning in mice lacking Sonic hedgehog gene function. Nature, 383(6599), 407-13 (1996)

30. S. C. Ekker, A. R. Ungar, P. Greenstein, D. P. von Kessler, J. A. Porter, R. T. Moon and P. A. Beachy: Patterning activities of vertebrate hedgehog proteins in the developing eye and brain. Curr Biol, 5(8), 944-55 (1995)
31. R. Macdonald, K. A. Barth, Q. Xu, N. Holder, I. Mikkola and S. W. Wilson: Midline signalling is required for Pax gene regulation and patterning of the eyes. Development, 121(10), 3267-78 (1995)

32. L. A. Schimmenti, J. De La Cruz, R. A. Lewis, J. D. Karkera, G. S. Manligas, E. Roessler and M. Muenke: Novel mutation in sonic hedgehog in non-syndromic colobomatous microphthalmia. Am J Med Genet, 116(3), 215-21 (2003)

33. R. O. Karlstrom, W. S. Talbot and A. F. Schier: Comparative synteny cloning of zebrafish you-too: mutations in the Hedgehog target gli2 affect ventral forebrain patterning. Genes Dev, 13(4), 388-393 (1999)

34. A. M. Barbieri, G. Lupo, A. Bulfone, M. Andreazzoli, M. Mariani, F. Fougerousse, G. G. Consalez, G. Borsani, J. S. Beckmann, G. Barsacchi, A. Ballabio and S. Banfi: A homeobox gene, vax2, controls the patterning of the eye dorsoventral axis. Proc Natl Acad Sci US A, 96(19), 10729-34 (1999)

35. S. H. Mui, R. Hinges, D. D. O'Leary, G. Lemke and S. Bertuzzi: The homeodomain protein Vax2 patterns the dorsoventral and nasotemporal axes of the eye. Development, 129, 797-804 (2002)

36. A. M. Barbieri, V. Broccoli, P. Bovolenta, G. Alfano, A. Marchitiello, C. Mocchetti, L. Crippa, A. Bulfone, V. Marigo, A. Ballabio and S. Banfi: Vax2 inactivation in mouse determines alteration of the eye dorsal-ventral axis, misrouting of the optic fibres and eye coloboma. Development, 129(3), 805-13 (2002)

37. A. Seth, J. Culverwell, M. Walkowicz, S. Toro, J. M. Rick, S. C. Neuhauss, Z. M. Varga and R. O. Karlstrom: belladonna/(Ihx2) is required for neural patterning and midline axon guidance in the zebrafish forebrain. Development, 133(4), 725-35 (2006)

38. E. Herrera, R. Marcus, S. Li, S. E. Williams, L. Erskine, E. Lai and C. Mason: Foxd1 is required for proper formation of the optic chiasm. Development, 131(22), 5727-39 (2004)

39. D. Schulte and C. L. Cepko: Two homeobox genes define the domain of EphA3 expression in the developing chick retina. Development, 127(23), 5033-45. (2000)

40. H. Takahashi, T. Shintani, H. Sakuta and M. Noda: CBF1 controls the retinotectal topographical map along the anteroposterior axis through multiple mechanisms. Development, 130(21), 5203-15 (2003)

41. R. C. Marcus, K. Shimamura, D. Sretavan, E. Lai, J. L. Rubenstein and C. A. Mason: Domains of regulatory gene expression and the developing optic chiasm: correspondence with retinal axon paths and candidate signaling cells. J Comp Neurol, 403(3), 346-358 (1999)

42. T. Pratt, M.-L Tiam, N., Simpson, T.I., Mason, J.O., Price, D.J: The winged helix transcription factor Foxg1 facilitates retinal ganglion cell axon crossing of the ventral midline in the mouse. Development, 131, 3773-3784 (2004) 43. K. Basler, T. Edlund, T. M. Jessell and T. Yamada: Control of cell pattern in the neural tube: regulation of cell differentiation by dorsalin-1, a novel TGF beta family member. Cell, 73(4), 687-702 (1993)

44. K. F. Liem, Jr., G. Tremml, H. Roelink and T. M. Jessell: Dorsal differentiation of neural plate cells induced by BMP-mediated signals from epidermal ectoderm. Cell, 82(6), 969-79 (1995) 
45. R. Rachel, J. Murdoch, F. Beermann, A. Copp and C. Mason: Retinal axon misrouting at the optic chiasm in mice with neural tube closure defects. Genesis, 27, 32-47 (2000)

46. Z. Kaprielian, E. Runko and R. Imondi: Axon guidance at the midline choice point. Dev Dyn, 221(2), 154-181. (2001)

K. Brose, K. S. Bland, K. H. Wang, D. Arnott, W. Henzel, C. S. Goodman, M. Tessier-Lavigne and T. Kidd: Slit proteins bind Robo receptors and have an evolutionarily conserved role in repulsive axon guidance. Cell, 96(6), 795-806. (1999)

47. T. Kidd, K. S. Bland and C. S. Goodman: Slit is the midline repellent for the robo receptor in Drosophila. Cell, 96(6), 785-94. (1999)

48. T. Kidd, K. Brose, K. J. Mitchell, R. D. Fetter, M. Tessier-Lavigne, C. S. Goodman and G. Tear: Roundabout controls axon crossing of the CNS midline and defines a novel subfamily of evolutionarily conserved guidance receptors. Cell, 92(2), 205-15 (1998)

49. E. Stein, Y. Zou, M. Poo and M. Tessier-Lavigne: Binding of DCC by netrin-1 to mediate axon guidance independent of adenosine A2B receptor activation. Science, 291(5510), 1976-82 (2001)

50. Y. Zou, E. Stoeckli, H. Chen and M. Tessier-Lavigne: Squeezing axons out of the gray matter: a role for slit and semaphorin proteins from midline and ventral spinal cord. Cell, 102(3), 363-75 (2000)

51. M. S. Deiner, T. E. Kennedy, A. Fazeli, T. Serafini, M. Tessier-Lavigne and D. W. Sretavan: Netrin-1 and DCC mediate axon guidance locally at the optic disc: loss of function leads to optic nerve hypoplasia. Neuron, 19(3), 575-589 (1997)

52. L. Erskine, S. E. Williams, K. Brose, T. Kidd, R. A. Rachel, C. S. Goodman, M. Tessier-Lavigne and C. A. Mason: Retinal ganglion cell axon guidance in the mouse optic chiasm: expression and function of robos and slits. $J$ Neurosci, 20(13), 4975-4982 (2000)

53. C. Fricke, J. S. Lee, S. Geiger-Rudolph, F. Bonhoeffer and C. B. Chien: astray, a zebrafish roundabout homolog required for retinal axon guidance. Science, 292(5516), 507-510 (2001)

54. L. D. Hutson and C. B. Chien: Pathfinding and error correction by retinal axons: the role of astray/robo2. Neuron, 33(2), 205-17. (2002)

55. A. S. Plump, L. Erskine, C. Sabatier, K. Brose, C. J. Epstein, C. S. Goodman, C. A. Mason and M. TessierLavigne: Slit1 and Slit2 cooperate to prevent premature midline crossing of retinal axons in the mouse visual system. Neuron, 33(2), 219-232 (2002)

56. K. Hannula-Jouppi, N. Kaminen-Ahola, M. Taipale, R. Eklund, J. Nopola-Hemmi, H. Kaariainen and J. Kere: The axon guidance receptor gene ROBO1 is a candidate gene for developmental dyslexia. PLoS Genet, 1(4), e50 (2005) 57. S. Nakagawa, C. Brennan, K. G. Johnson, D. Shewan, W. A. Harris and C. E. Holt: Ephrin-B regulates the Ipsilateral routing of retinal axons at the optic chiasm. Neuron, 25(3), 599-610 (2000)

58. S. E. Williams, F. Mann, L. Erskine, T. Sakurai, S. Wei, D. J. Rossi, N. W. Gale, C. E. Holt, C. A. Mason and M. Henkemeyer: Ephrin-B2 and EphB1 mediate retinal axon divergence at the optic chiasm. Neuron, 39(6), 919-35 (2003)
S. E. Williams, C. Mason and E. Herrera: The optic chiasm as a midline choice point. Curr. Op. Neurobiol., 14:51-60 (2004)

59. M. A. Lambot, F. Depasse, J. C. Noel and P. Vanderhaeghen: Mapping labels in the human developing visual system and the evolution of binocular vision. $J$ Neurosci, 25(31), 7232-7 (2005)

60. E. Herrera, L. Y. Brown, J. Aruga, R. A. Rachel, G. Dolen, K. Mikoshiba, S. Brown and C. A. Mason: Zic2 patterns binocular vision by specifying the uncrossed retinal projection. Cell, 114(5), 545-557 (2003)

61. S. A. Brown, D. Warburton, L. Y. Brown, C. Y. Yu, E. R. Roeder, S. Stengel-Rutkowski, R. C. Hennekam and M. Muenke: Holoprosencephaly due to mutations in ZIC2, a homologue of Drosophila odd-paired. Nat Genet, 20(2), 180-3 (1998)

62. W. Pak, R. Hindges, Y. S. Lim, S. L. Pfaff and D. O'Leary D: Magnitude of Binocular Vision Controlled by Islet-2 Repression of a Genetic Program that Specifies Laterality of Retinal Axon Pathfinding. Cell, 119(4), 56778 (2004)

63. K. Y. Chung: Regionally specific expression of L1 and sialylated NCAM in the retinofugal pathway of mouse embryos. J Comp Neurol, 471(4), 482-98 (2004)

64. S. E. Williams, M. Grumet, D. R. Colman, M. Henkemeyer, C. A. Mason and T. Sakurai: A role for NrCAM in the patterning of binocular visual pathways. Neuron, 50(4), 535-47 (2006)

Key Words: Optic chiasm, Retina, Axon Guidance, Review

Send correspondence to: Dr Eloisa Herrera, Instituto de Neurociencias de Alicante, Universidad Miguel HernandezCSIC, Campus de San Juan, Apt 18, San Juan de Alicante, Alicante 03550, Spain, Tel: 34-965-919231, Fax: 34-965919561, E-mail: e.herrera@umh.es

http://www.bioscience.org/current/vol13.htm 